\title{
Nuclear Modification of Two-hadron Correlations from HERMES
}

\author{
N.Bianchi for the HERMES Collaboration
}

INFN Laboratori Nazionali di Frascati, I-00044, Frascati, Italy.

\begin{abstract}
Semi-Inclusive DIS (SIDIS) of high energy leptons on nuclear targets has been used to study the nuclear medium modification of the quark fragmentation function. The possible interpretations and the implications on the parton energy loss are discussed. The first measurement of double-hadron production in deep-inelastic scattering within the nuclear medium is also presented. Nuclear effects on the additional hadron are clearly observed, but with little or no difference among nitrogen, krypton or xenon, and with smaller magnitude than effects seen on previously measured single-hadron multiplicities. The data are compared with models based on partonic energy loss or pre-hadronic scattering, and with a model based on a purely absorptive treatment of the final state interactions. Thus, the double-hadron ratio provides an additional tool for studying modifications of hadronization in nuclear matter.
\end{abstract}

\section{Introduction}

Hadron production from a free nucleon in deep-inelastic scattering is generally described by fragmentation functions that contain non-perturbative information about parton hadronization. These functions are expected to be different for nuclear targets [1] because of several possible effects: energy loss of the propagating quarks, rescattering during the pre-hadronic formation process or interactions of the final-state hadrons within the nucleus. Despite recent accurate experimental data from single-hadron leptoproduction [2] the underlying mechanisms in theoretical models for hadronization in the nuclear medium differ greatly [3-7]. Both partonic and hadronic interpretations have been advocated.

Double-hadron leptoproduction offers an additional way to study hadronization. If partonic energy loss of the struck quark were the only mechanism involved, it would be naïvely expected that the attenuation effect does not depend strongly on the number of hadrons involved, and the double-hadron to 
single-hadron ratio for a nuclear target should be only slightly dependent on the mass number $A$. On the contrary, if final hadron absorption were the dominant process, the requirement of an additional slower sub-leading hadron that is more strongly absorbed would suppress the two-hadron yield from heavier nuclei $[5,8]$, so that this ratio should decrease with $A$.

\section{Fragmentation functions and multiplicities}

The SIDIS process can be factorized in a parton distribution function $q_{f}$, which is function of the Bjorken scaling variable $x=Q^{2} /(2 M \nu)$, a hard scattering cross section $d \sigma_{f}$ calculable in QCD, and a parton fragmentation function $D_{f}^{h}$ which is function of the fractional $z=E_{h} / \nu$ of the energy of the struck quark. In the Quark Parton Model (QPM) description of SIDIS the fragmentation functions are multiplied by the parton distributions, and experimental hadron multiplicities $M^{h}$ are determined by normalizing the SIDIS yield to the DIS rate:

$$
M^{h}=\frac{1}{N_{D I S}} \frac{d N^{h}(x, z)}{d z}=\frac{\sum_{f} e_{f}^{2} q_{f}(x) D_{f}^{h}(z)}{\sum_{f} e_{f}^{2} q_{f}(x)}
$$

It is easy to demonstrate that within the QPM and under the $u$-quark dominance assumption, the pion multiplicities are almost equivalent to the pion fragmentation functions. In QCD the relation between fragmentation functions and multiplicities is more complicated since both the leading order (LO) and the next to leading order (NLO) terms of the cross section are convoluted with parton distributions and with the hadron fragmentation functions. Nevertheless, it has been shown [9] that, once integrating over a broad range of $x$, the SIDIS multiplicities are in good agreement with the NLO-QCD evolution of fragmentation functions determined from $e^{+} e^{-}$measurements at LEP. This result suggests the validity of the factorization assumption for the SIDIS process and of the approximation of fragmentation functions with multiplicities.

\section{Hadronization in the nuclear medium}

The study of the medium modification of fragmentation functions can be performed by SIDIS measurements on nuclear targets where the nucleus acts as an ensemble of targets which reduce the multiplicity of fast hadrons due to both partonic and hadronic interactions. The recent results from HERMES [2] provided a new strong boost of interest in this field. HERMES [10] is an experiment at DESY which is mainly devoted to the study of the spin structure 


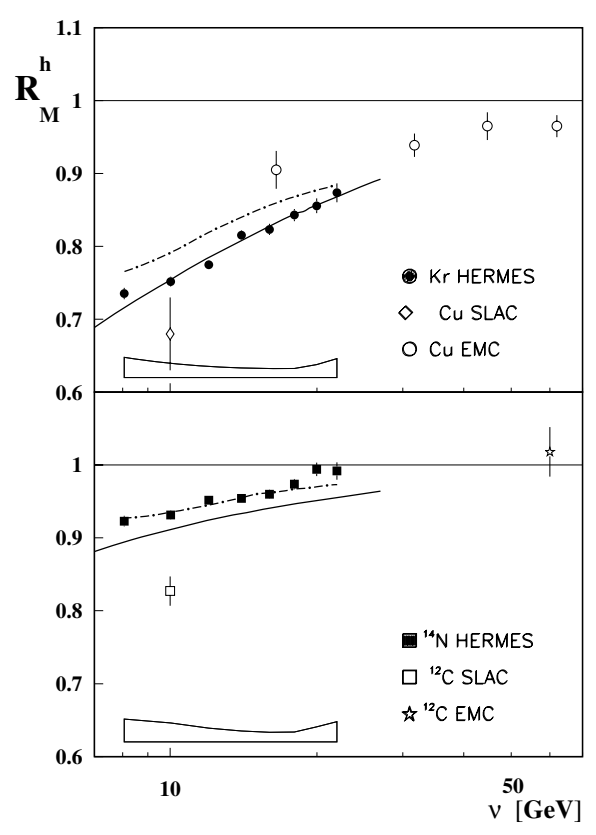

Fig. 1. Multiplicity ratios as a function of $\nu$ for hadrons with $z>0.2$ for HERMES, SLAC and EMC. The solid curves are calculations from [7] and the dot-dashed curves are calculations from [3]

of the nucleon by using a polarized positron (or electron) beam and polarized internal gas targets. In addition, some HERMES runs have been performed with unpolarized nuclear gas targets (D, $\left.{ }^{4} \mathrm{He}, \mathrm{N}, \mathrm{Ne}, \mathrm{Kr}, \mathrm{Xe}\right)$ with densities up to $\sim 10^{16} \mathrm{nucl} \cdot \mathrm{cm}^{-2}$, allowing the extension of the physics program to the study of several nuclear effects.

In fig. 1 the HERMES results for the fast hadron multiplicity ratios from $\mathrm{N}$ and $\mathrm{Kr}$ relative to that from deuterium, are shown in comparison with data from earlier experiments and with theoretical predictions. As it is seen, the HERMES kinematic is well suited to study quark propagation and hadronisation, and the results indicate an increase of the multiplicity ratio (thus a decrease of the medium effect) with $\nu$, in agreement with the higher energy EMC data.

The complete particle identification in HERMES allows to fully disentangle the information for different hadron types. This is shown in Fig. 2 where the multiplicity ratios between $\mathrm{Kr}$ and D nuclei are presented as function of the scaling variable $z$. As it is seen, the medium effects for $\pi^{+}$and $\pi^{-}$are equal. Also the medium effects on $K^{-}$production are similar to the charged pion case. Quite interesting is the difference between $K^{+}$and $K^{-}$and especially between $p$ and $\bar{p}$. In particular the effect for the proton is strongly different at low- 


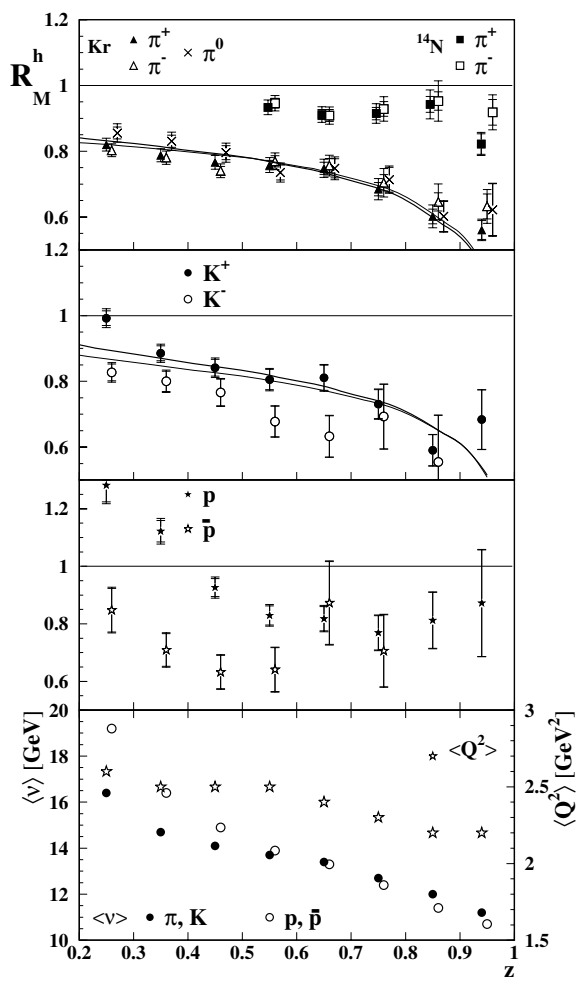

Fig. 2. HERMES multiplicity ratio Kr/D for different charged hadrons as a function of the scaling variable $z$. In the lower panel the relevant average kinematic variables are presented for $z$-bin. The thick (thin) solid curves represent the calculations from [7] for positive (negative) charge states.

$z$, where a contamination from the target fragmentation can contribute. The different results for different hadrons may reveal differences in the modification of $q$ and $\bar{q}$ fragmentation functions, in the hadron formation times and in the different hadronic interaction cross sections.

New preliminary HERMES results obtained on different nuclei suggest a dependence of the nuclear attenuation $\left(1-R_{A}^{\pi}\right) \approx A^{2 / 3}$, i.e. proportional to the square of the nuclear size. A decreasing nuclear effect with the increasing $Q^{2}$ can be also derived from these preliminary data.

\section{Modification of fragmentation functions}

The new HERMES results have been interpreted with theoretical models describing hadron production on nuclei in terms of a modification of the quark fragmentation functions. 
In Ref. [3] the modification of quark fragmentation functions and their QCD evolution are described in the framework of multiple parton scattering. As consequence of the partonic energy loss $d E / d x$ due to the induced gluon radiation, the fragmentation function in the nucleus $D^{A}\left(z, Q^{2}\right)$ is modified compared to the one in deuterium $D^{D}\left(z, Q^{2}\right)$. In the framework of this calculation, for a cold and static system like the nucleus, the quark energy loss involved in SIDIS reaction has been determined. The extracted energy loss is $d E / d x \approx 0.5$ $\mathrm{GeV} / \mathrm{fm}$ for a quark with $\mathrm{E}=10 \mathrm{GeV}$ in an $\mathrm{Au}$ nucleus. Including the effect of the expansion, an increase of the gluon density of a factor $\sim 30$ has been derived for the initial hot stage of $A u+A u$ collisions at RHIC at $\sqrt{s}=130$ $\mathrm{GeV}$. A theoretical description of the HERMES data in terms of in-medium distribution of the parton energy loss has been also given in Ref. [4]. The calculation quite well reproduce the HERMES data, and the derived energy loss per unit length $d E / d x=0.62 \mathrm{GeV} / \mathrm{fm}$ is close to the result of Ref. [3].

In Ref. [5] the combined effect of the fragmentation function modification due to the induced gluon radiation and of the hadron interaction inside the nucleus has been calculated. The contribution due to the interaction of the pre-formed hadron in the nuclear medium was claimed to be large.

Also in Ref. [7] the nuclear absorption of the pre-formed hadrons is seen to significantly affect the hadron production on heavy nuclei in the kinematic region of the HERMES experiment. In this work the modification of quark fragmentation functions have been considered in terms of $Q^{2}$-rescaling model, originally developed to interpret the EMC-effect in the nuclear structure functions, and of absorption of the produced hadrons. The deconfinement scale is assumed to be proportional to the degree of overlap of the nucleons inside the given nucleus.

In Ref. [6] the data analysis was carried out in the framework of a probabilistic coupled-channel transport model which allowed for a treatment of the final-state interactions beyond simple absorption mechanisms. Furthermore, the event by event simulations accounted for the kinematics cuts of the experiment as well as the geometrical acceptance of the detectors. This study suggested that the pre-hadronic final state interactions play a dominant role in the kinematic regime of HERMES while the approach overestimate the small attenuation observed at EMC energies.

\section{Double-hadron leptoproduction}

In double-hadron leptoproduction, semi-inclusive deep-inelastic scattering data are presented in terms of the ratio 


$$
R_{2 h}\left(z_{2}\right)=\frac{\left(\frac{d N^{z_{1}>0.5}\left(z_{2}\right) / d z_{2}}{N^{z_{1}>0.5}}\right)_{A}}{\left(\frac{d N^{z_{1}>0.5}\left(z_{2}\right) / d z_{2}}{N^{z_{1}>0.5}}\right)_{D}}
$$

The values $z_{1}$ and $z_{2}$ correspond to the leading and sub-leading hadrons, respectively. The quantity $d N^{z_{1}>0.5}$ is the number of events with at least two detected hadrons in a bin of width $d z_{2}$ at $z_{2}$ with $z_{1}>0.5$. The quantity $N^{z_{1}>0.5}$ is the number of events with at least one detected hadron with $z_{1}>0.5$. Two methods of double-hadron event selection were used. Selection I contains only the combinations of hadron charges (leading-subleading) $++,--,+0,0+,-0$, $0-, 00$. This suppresses the contributions from $\rho^{0} \rightarrow \pi^{+} \pi^{-}$decay because the +- and -+ combinations are missing. Moreover, in the Lund string model, the exclusion of the opposite-charge combinations enhances the rank-1 (leading) plus rank-3 (sub-leading) combination. The higher the particle rank, the more likely it is formed deep inside the nucleus, and the corresponding hadron absorption should be larger. Selection II contains all particle charge combinations. Here, the sub-leading hadron is mainly of rank-2 and the contribution from $\rho^{0}$ decay is larger.

Fig. 3 shows the double ratio $R_{2 h}$ as a function of $z_{2}$ for Selection I only [14]. The kinematic variables are in the range $\langle\nu\rangle=21$ to $16 \mathrm{GeV}$ and $\left\langle Q^{2}\right\rangle=2.1$ to $2.6 \mathrm{GeV}^{2}$ as $z_{2}$ goes from 0.09 to 0.44 . The averages over $z_{2}$ are $\langle\nu\rangle=17.7$ $\mathrm{GeV}$ and $\left\langle Q^{2}\right\rangle=2.4 \mathrm{GeV}^{2}$. The ratio $R_{2 h}$ is generally below unity with no significant difference between the three nuclei. These data clearly show that the nuclear effect in the double-hadron ratio is much smaller than for the single-hadron attenuation measured under the same kinematic conditions. For $z_{2}<0.1$, where $R_{2 h}$ rises towards and possibly above 1 , the slow hadrons originate largely from target fragmentation [6,13]. Also for $z_{2}>0.4$, where the two hadrons have similar energy, $R_{2 h}$ seems to rise towards unity. Fig. 3 (upper panel) shows calculations based on a PYTHIA event generator with a fully coupled-channel treatment of final-state interactions by means of a BUU transport model [6]. In this model, the fragmentation function is modified by pre-hadron interactions and rescattering in the medium. Although the general trend of the data is reproduced, the model predicts an effect twice as large for xenon and krypton as for nitrogen above $z_{2}=0.1$, which is not supported by the data. Fig. 3 (bottom panel) shows the same data compared to a calculation with a purely absorptive treatment of the interaction of the pre-hadronic or the final hadronic states. The data rule out this assumption [6].

Fig. 4 presents $R_{2 h}$ calculated for all hadron charge combinations (Selection II) [14]. Inclusion of the +- and -+ pairs does not change the value of $R_{2 h}$ significantly, contrary to all of the naïve space-time evolutionary models of hadronization. In order to evaluate further the possible influence of the exclusive and semi-inclusive $\rho^{0}$ production, $R_{2 h}$ was extracted for all hadron pairs except those with invariant mass near the $\rho^{0}$. This has no noticeable 


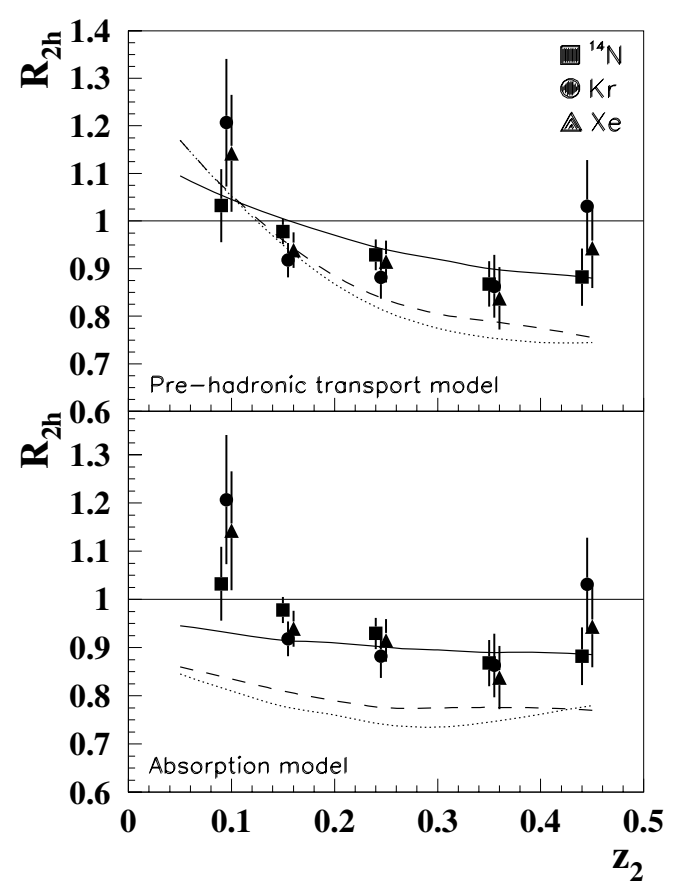

Fig. 3. The ratio $R_{2 h}$ as a function of $z_{2}$ for ${ }^{14} \mathrm{~N}$ (squares), $\mathrm{Kr}$ (circles) and Xe (triangles) with $z_{1}>0.5$. Only Selection I was considered. The systematical uncertainty is $2 \%$ for all the targets and is independent of $z_{2}$. In the upper panel the curves (solid for ${ }^{14} \mathrm{~N}$, dashed for $\mathrm{Kr}$, dotted for $\mathrm{Xe}$ ) are calculated within a BUU transport model [6]. In the bottom panel the same data are shown with calculations that assume only absorption for the three nuclei (same line types as in the upper plot) $[6]$.

effect on $R_{2 h}$. Therefore, the final data are presented over the full invariant mass range. The effect of only the exclusive $\rho^{0}$ production is even smaller since it contributes only $5 \%$ of the total yield.

The curves in Fig. 4 represent the model [11,12] in which modifications of the fragmentation functions arises from parton energy loss. Contrary to naïve expectations, this model predicts a significant $A$-dependence, in conflict with the data.

\section{References}

[1] M. Gyulassy and M. Plumer, Nucl. Phys. B 346, 1 (1990).

[2] HERMES Coll., A. Airapetian et al.; Phys. Lett. B 577, 37 (2003), Eur. Phys. J. C 20, 479 (2001). 


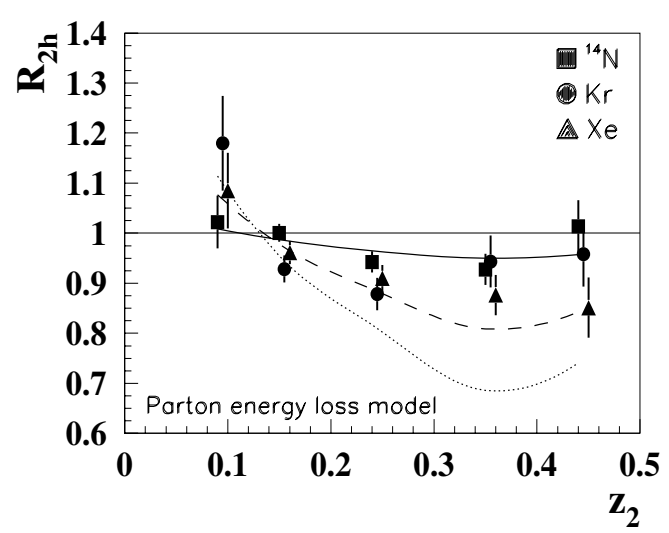

Fig. 4. The ratio $R_{2 h}$ as a function of $z_{2}$ for ${ }^{14} \mathrm{~N}$ (squares), Kr (circles) and Xe (triangles) with $z_{1}>0.5$ for Selection II. The systematic uncertainty is $4 \%$ (3\%) for xenon and krypton (nitrogen) and is independent of $z_{2}$. The curves $\left({ }^{14} \mathrm{~N}\right.$ : solid; Kr: dashed; Xe: dotted) are from the parton energy loss model described in Refs.[11,12].

[3] X.-N. Wang and X. Guo, Nucl. Phys. A 696, 788 (2001); E. Wang and X.N. Wang, Phys. Rev. Lett. 89, 162301 (2002).

[4] F. Arleo, Nucl. Phys. A 715, 899 (2003).

[5] B. Z. Kopeliovich et al., Nucl. Phys. A 740, 211 (2004).

[6] T. Falter et al., Phys. Lett. B 594, 61 (2004); Phys. Rev. C 70, 054609 (2004); K. Gallmeister and W. Cassing, Nucl. Phys. A 748, 241 (2005).

[7] A. Accardi et al., Nucl. Phys. A 720, 131 (2003); A. Accardi et al. Nucl. Phys. A 761, 67 (2005).

[8] X.-N. Wang, Phys. Lett. B 595, 165 (2004); A. Majumder and X.-N. Wang, Phys. Rev. D 70, 014007 (2004); X.-N. Wang, Nucl. Phys. A 750, 98 (2005).

[9] HERMES Coll., A. Airapetian et al., Eur. Phys. J. C 21 (2001) 599.

[10] HERMES Coll., K. Ackerstaff et al., Nucl. Instr. and Meth. A 417, 230 (1998).

[11] X.-N. Wang, Phys. Lett. B 579, 299 (2004).

[12] A. Majumder, Eur. Phys. J. C 43, 259 (2005); Private Communication.

[13] K. Gallmeister and T. Falter, Phys. Lett. B 630, 40 (2005).

[14] HERMES Coll., A. Airapetian et al., Phys. Rev. Lett. 96, 162301 (2006). 